\title{
Cyclic hybrid methods for finding common fixed points of a finite family of nonexpansive mappings
}

\author{
Qiao-Li Donga,b, Yan-Yan Lu ${ }^{a}$, Jinfeng Yang ${ }^{b, *}$ \\ ${ }^{a}$ College of Science, Civil Aviation University of China, Tianjin 300300, China. \\ ${ }^{b}$ Tianjin Key Lab for Advanced Signal Processing, Civil Aviation University of China, Tianjin 300300, China. \\ Communicated by Y. Yao
}

\begin{abstract}
In this paper, we propose a cyclic hybrid method for computing a common fixed point of a finite family of nonexpansive mappings. The strong convergence of the method is established. Numerical examples illustrate that the proposed method has an advantage in computing. (c)2016 All rights reserved.
\end{abstract}

Keywords: Common fixed point, hybrid method, cyclic computation, nonexpansive mapping. 2010 MSC: 47H05, 47H07, 47H10.

\section{Introduction and Preliminaries}

Let $H$ be a real Hilbert space with inner product $\langle\cdot, \cdot\rangle$ and norm $\|\cdot\|$ and $C$ a nonempty closed convex subset of $H$. Recall that a mapping $T: C \rightarrow C$ is said to be nonexpansive if

$$
\|T x-T y\| \leq\|x-y\|
$$

holds for all $x, y \in C$. We denote by Fix $(T)$ the set of fixed points of $T$, i.e., $F i x(T)=\{x \in C: T x=x\}$.

Construction of common fixed points for a finite family of nonexpansive mappings have received vast investigation, see [3, 6, 13, 16], since various problems of science and engineering, such as split feasibility problems and multiple-sets split feasibility problems whit applications in intensity-modulated radiation therapy (IMRT) in the field of medical care, see [4, 5], can be reduced to a problem of finding a common fixed point of a family of nonexpansive mappings.

\footnotetext{
${ }^{*}$ Corresponding author

Email address: jfyang@cauc.edu.cn (Jinfeng Yang)
} 
In 2003, Nakajo and Takahashi [12] firstly introduced a hybrid algorithm for a nonexpansive mapping, thereafter, several researchers generalized the hybrid methods for computing common fixed points of a family of nonlinear mappings, see [7, 8, 14, 17, 18, 21]. For a finite family of relatively nonexpansive mappings $\left\{T_{i}\right\}_{i=1}^{N}$, Anh and Chung [1] recently proposed a parallel hybrid algorithm as following:

\section{Algorithm AC}

$$
\left\{\begin{array}{l}
x_{0} \in C \quad \text { chosen arbitrarily, } \\
z_{k}:=P_{C}\left(x_{k}\right), \\
y_{k}^{i}:=\alpha_{k} z_{k}+\left(1-\alpha_{k}\right) T_{i}\left(z_{k}\right), \quad i=1,2, \cdots, N, \\
i_{k}:=\underset{i=1,2, \cdots, N}{\operatorname{argmax}}\left\{\left\|y_{k}^{i}-x_{k}\right\|\right\}, \\
C_{k}:=\left\{v \in H:\left\|v-y_{k}^{i_{k}}\right\| \leq\left\|v-x_{k}\right\|\right\}, \\
Q_{k}:=\left\{u \in H:\left\langle x_{0}-x_{k}, x_{k}-u\right\rangle \geq 0\right\}, \\
x_{k+1}:=P_{C_{k} \cap Q_{k}}\left(x_{0}\right) .
\end{array}\right.
$$

Algorithm AC is inherently parallel and Anh and Chung showed their advantage in parallel computation in numerical examples.

Motivating by Anh and Chung's work, we proposed a cyclic hybrid method which can be regarded as a counterpart of the parallel one. Our ideas consists of determining successively $y_{k}^{i}$ for each operator $T_{i}$, $i=1,2, \ldots, N$ and constructing of $y_{k}^{i}$ by using the value of $y_{k}^{i-1}$. Subsequent steps are the same with Algorithm AC. The benefit of our approach is using the newly-obtained $y_{k}^{i-1}\left(y_{k}^{0}=x_{k}\right)$.

The remainder of this article is organized as follows. In the next section, some useful facts and tools are given. Convergence analysis of the cyclic algorithm is given in Section 3 , while in Section 4 the numerical experiment is considered.

\section{Preliminaries}

We will use the notation:

1. $\rightarrow$ for weak convergence and $\rightarrow$ for strong convergence.

2. $\omega_{w}\left(x_{n}\right)=\left\{x: \exists x_{n_{j}} \rightarrow x\right\}$ denotes the weak $\omega$-limit set of $\left\{x_{n}\right\}$.

We need some facts and tools in a real Hilbert space $H$ which are listed as lemmas below.

Lemma 2.1 ([2]). There holds the identity in a real Hilbert space $H$ :

$$
\|u-v\|^{2}=\|u\|^{2}-\|v\|^{2}-2\langle u-v, v\rangle, \quad u, v \in H .
$$

Lemma 2.2 ([10]). Let $C$ be a closed convex subset of a real Hilbert space $H$ and let $T: C \rightarrow C$ be a nonexpansive mapping such that Fix $(T) \neq \emptyset$. If a sequence $\left\{x_{n}\right\}$ in $C$ is such that $x_{n} \rightarrow z$ and $x_{n}-T x_{n} \rightarrow 0$, then $z=T z$.

Lemma 2.3 ([2]). Let $K$ be a closed convex subset of real Hilbert space $H$ and let $P_{K}$ be the (metric or nearest point) projection from $H$ onto $K$ (i.e., for $x \in H, P_{K} x$ is the only point in $K$ such that $\left\|x-P_{K} x\right\|=$ $\inf \{\|x-z\|: z \in K\})$. Given $x \in H$ and $z \in K$. Then $z=P_{K} x$ if and only if there holds the relation:

$$
\langle x-z, y-z\rangle \leq 0, \quad \text { for all } y \in K .
$$

Lemma 2.4 ([11]). Let $K$ be a closed convex subset of $H$. Let $\left\{x_{n}\right\}$ be a sequence in $H$ and $u \in H$. Let $q=P_{K} u$. If $\left\{x_{n}\right\}$ is such that $\omega_{w}\left\{x_{n}\right\} \subset K$ and satisfies the condition

$$
\left\|x_{n}-u\right\| \leq\|u-q\|, \quad \text { for all } n
$$

then $x_{n} \rightarrow q$. 


\section{A cyclic hybrid algorithm and its convergence}

Let $\left\{T_{i}\right\}_{i=1}^{N}$ be a family of nonexpansive mappings from $C$ into itself and assume that the set $F:=$ $\bigcap_{i=1}^{N} F\left(T_{i}\right)$ is not empty.

We consider the following algorithm.

Algorithm 3.1. Let $x_{0} \in C$ be an arbitrarily chosen element and $\left\{\alpha_{k}\right\} \subset(0, \alpha]$ where $\alpha<1$. For $k \geq 0$, assuming $x_{k}$ is known, we

-Calculate

$$
\left\{\begin{aligned}
y_{k}^{1} & :=\alpha_{k} x_{k}+\left(1-\alpha_{k}\right) T_{1}\left(x_{k}\right), \\
y_{k}^{i+1} & :=\alpha_{k} y_{k}^{i}+\left(1-\alpha_{k}\right) T_{i+1}\left(y_{k}^{i}\right), \quad i=0,1, \cdots, N-1 .
\end{aligned}\right.
$$

$\bullet$ Find

$$
i_{k}:=\underset{i=1,2, \cdots, N}{\operatorname{argmax}}\left\{\left\|y_{k}^{i}-x_{k}\right\|\right\}
$$

-If $\left\|y_{k}^{i_{k}}-x_{k}\right\|=0$ then stop. Else:

-Define

$$
\left\{\begin{array}{l}
C_{k}:=\left\{u \in C:\left\|u-y_{k}^{i_{k}}\right\| \leq\left\|u-x_{k}\right\|\right\} \\
Q_{k}:=\left\{v \in C:\left\langle x_{0}-x_{k}, x_{k}-v\right\rangle \geq 0\right\} .
\end{array}\right.
$$

-Compute

$$
x_{k+1}:=P_{C_{k}} \cap Q_{k}\left(x_{0}\right) .
$$

-If $x_{k+1}=x_{k}$ then stop. Else, set $\mathrm{k}:=\mathrm{k}+1$ and repeat.

Lemma 3.2. If Algorithm 3.1 finishes at a step $k<\infty$, then $x_{k}$ is a common fixed point of $T_{i}, i=1,2, \ldots, N$, i.e., $x_{k} \in$ Fix $\left(T_{i}\right)$.

Proof. Using stopping rule $x_{k}=x_{k+1}$, we have $x_{k} \in C_{k}$. From the definition of $C_{k}$, it follows

$$
\left\|x_{k}-y_{k}^{i_{k}}\right\| \leq\left\|x_{k}-x_{k}\right\|=0
$$

Applying the definition of $i_{k}$, we get $y_{k}^{i}=x_{k}$ for $i=1,2, \ldots, N$. Taking into account (3.1), we have

$$
x_{k}=\alpha_{k} x_{k}+\left(1-\alpha_{k}\right) T_{i}\left(x_{k}\right), \quad i=1,2, \cdots, N .
$$

Since $\alpha_{k}<1$ we see $x_{k}=T_{i}\left(x_{k}\right)$ for $i=1,2, \cdots, N$, i.e., $x_{k} \in \operatorname{Fix}\left(T_{i}\right)$.

Theorem 3.3. Let $\left\{x_{k}\right\}$ be the (infinite) sequence generated by Algorithm 3.1, $T_{i}$ be nonexpansive for $i=1,2, \ldots, N$. Then $x_{k} \rightarrow x^{\dagger}:=P_{F i x\left(T_{i}\right)}\left(x_{0}\right)$ as $k \rightarrow \infty$.

Proof. For each $k \geq 0$, it is easy to see that $Q_{k}$ is a halfspace or $Q_{k}=H$. Further, the relation $\left\|u-y_{k}^{i_{k}}\right\| \leq$ $\left\|u-x_{k}\right\|$ is equivalent to $\left\langle u, x_{k}-y_{k}^{i_{k}}\right\rangle \leq \frac{1}{2}\left\{\left\|x_{k}\right\|^{2}-\left\|y_{k}^{i_{k}}\right\|^{2}\right\}$ or $\left\langle u-\frac{1}{2}\left(x_{k}+y_{k}^{i_{k}}\right), x_{k}-y_{k}^{i_{k}}\right\rangle \leq 0$. Hence, for all $k \geq 0, C_{k}$ is a halfspace in $H$ or $C_{k}=H$. An explicit formula for $P_{C_{k}} \cap Q_{k}\left(x_{0}\right)$ can be obtained similarly as in [15]. Therefore, if $C_{k} \cap Q_{k} \neq \varnothing$ then $x_{k+1}$ is easily computed by (3.4).

Next we show that $\operatorname{Fix}\left(T_{i}\right) \subset C_{k} \bigcap Q_{k}$. Firstly we show that $F i x\left(T_{i}\right) \subset C_{k}$ for all $k \geq 0$. To observe this, arbitrarily take $p \in \operatorname{Fix}\left(T_{i}\right)$, we have

$$
\left\|p-y_{k}^{i_{k}}\right\|=\left\|p-\left\{\alpha_{k} y_{k}^{i-1}+\left(1-\alpha_{k}\right) T_{i}\left(y_{k}^{i-1}\right)\right\}\right\|
$$




$$
\begin{aligned}
& \leq \alpha_{k}\left\|p-y_{k}^{i-1}\right\|+\left(1-\alpha_{k}\right)\left\|p-T_{i}\left(y_{k}^{i-1}\right)\right\| \\
& \leq \alpha_{k}\left\|p-y_{k}^{i-1}\right\|+\left(1-\alpha_{k}\right)\left\|p-y_{k}^{i-1}\right\| \\
& =\left\|p-y_{k}^{i-1}\right\| \\
& \leq \cdots \leq\left\|p-y_{k}^{1}\right\| \leq\left\|p-x_{k}\right\| .
\end{aligned}
$$

Therefore, $p \in C_{k}$ and, hence, Fix $\left(T_{i}\right) \subset C_{k}$ for all $k \geq 0$.

Next we show Fix $\left(T_{i}\right) \subset Q_{k}$ for all $k \geq 0$, by induction. For $k=0$, we have Fix $\left(T_{i}\right) \subset C=Q_{0}$. Assume Fix $\left(T_{i}\right) \subset Q_{k}$. Since $x_{k+1}$ is the projection of $x_{0}$ onto $C_{k} \bigcap Q_{k}$, by Lemma 2.3, we have $\left\langle x_{0}-x_{k}, x_{k}-u\right\rangle \geq 0$, for $u \in C_{k} \cap Q_{k}$. As $F i x\left(T_{i}\right) \subset C_{k} \cap Q_{k}$, by the induction assumption, the last inequality holds, in particular, for all $u \in F i x\left(T_{i}\right)$. This together with the definition of $Q_{k+1}$ implies that Fix $\left(T_{i}\right) \subset Q_{k+1}$. Therefore we have $\operatorname{Fix}\left(T_{i}\right) \subset Q_{k}$ for all $k \geq 0$. Hence $\operatorname{Fix}\left(T_{i}\right) \subset C_{k} \bigcap Q_{k}$.

Since Fix $\left(T_{i}\right)$ is a nonempty closed convex subset of $C$, there exists a unique element $x^{\dagger} \in F i x\left(T_{i}\right)$ such that $x^{\dagger}=P_{F i x\left(T_{i}\right)} x_{0}$. From $x_{k}=P_{Q_{k}} x_{0}$ (by the definition of $Q_{k}$ ) and Fix $\left(T_{i}\right) \subset Q_{k}$, we have $\left\|x_{k}-x_{0}\right\| \leq\left\|p-x_{0}\right\|$ for all $p \in \operatorname{Fix}\left(T_{i}\right)$. Due to $x^{\dagger} \in$ Fix $\left(T_{i}\right)$, then we get

$$
\left\|x_{k}-x_{0}\right\| \leq\left\|x^{\dagger}-x_{0}\right\|
$$

which implies that $\left\{x_{k}\right\}$ is bounded.

The fact that $x_{k+1} \in Q_{k}$ implies that $\left\langle x_{k+1}-x_{k}, x_{k}-x_{0}\right\rangle \geq 0$. This together with Lemma 2.1 imply

$$
\left\|x_{k+1}-x_{k}\right\|^{2} \leq\left\|x_{k+1}-x_{0}\right\|^{2}-\left\|x_{k}-x_{0}\right\|^{2} .
$$

From (3.5) and (3.6) we obtain

$$
\left\|x_{k}-x_{k+1}\right\| \rightarrow 0 \quad \text { as } k \rightarrow \infty .
$$

Using the definition of $C_{k}$ and the inclusion $x_{k+1} \in C_{k}$, we also have

$$
\left\|x_{k+1}-y_{k}^{i_{k}}\right\| \leq\left\|x_{k+1}-x_{k}\right\|
$$

which with (3.7) yields

$$
\begin{aligned}
\left\|x_{k}-y_{k}^{i_{k}}\right\| & \leq\left\|x_{k+1}-x_{k}\right\|+\left\|x_{k+1}-y_{k}^{i_{k}}\right\| \\
& \leq 2\left\|x_{k+1}-x_{k}\right\| \rightarrow 0 \text { as } k \rightarrow \infty .
\end{aligned}
$$

From the definition of $i_{k}$ in $(3.2)$, it follows that

$$
\left\|x_{k}-y_{k}^{i}\right\| \rightarrow 0 \quad \text { as } k \rightarrow \infty \quad \text { for } i=1,2, \cdots, N,
$$

which implies

$$
\left\|y_{k}^{i+1}-y_{k}^{i}\right\| \leq\left\|y_{k}^{i+1}-x_{k}\right\|+\left\|y_{k}^{i}-x_{k}\right\| \rightarrow 0, \quad \text { for } i=1,2, \cdots, N-1 .
$$

From (3.1) it follows

$$
\left\|x_{k}-T_{1}\left(x_{k}\right)\right\|=\frac{1}{1-\alpha_{k}}\left\|y_{k}^{1}-x_{k}\right\| \rightarrow 0
$$

and

$$
\left\|y_{k}^{i}-T_{i+1}\left(y_{k}^{i}\right)\right\|=\frac{1}{1-\alpha_{k}}\left\|y_{k}^{i}-y_{k}^{i+1}\right\| \rightarrow 0, \quad i=1,2, \ldots, N-1 .
$$

Using (3.8), 3.10 and nonexpansivity of $\left\{T_{i}\right\}_{i=2}^{N}$, we get

$$
\begin{aligned}
\left\|x_{k}-T_{i+1}\left(x_{k}\right)\right\| & \leq\left\|x_{k}-y_{k}^{i}\right\|+\left\|y_{k}^{i}-T_{i+1}\left(y_{k}^{i}\right)\right\|+\left\|T_{i+1}\left(x_{k}\right)-T_{i+1}\left(y_{k}^{i}\right)\right\| \\
& \leq 2\left\|x_{k}-y_{k}^{i}\right\|+\left\|y_{k}^{i}-T_{i+1}\left(y_{k}^{i}\right)\right\| \rightarrow 0, \quad i=1,2, \ldots, N-1 .
\end{aligned}
$$

Equations (3.9), (3.11) and Lemma 2.2 imply that $\omega_{w}\left(x_{k}\right) \subset F i x\left(T_{i}\right), i=1,2, \ldots, N$, i.e., $\omega_{w}\left(x_{k}\right) \subset F$. This, together with (3.5) and Lemma 2.4, guarantee strong convergence of $x_{k}$ to $P_{F i x\left(T_{i}\right)} x_{0}$. 


\section{A numerical example}

In this section, we perform Algorithm 3.1 and Algorithm AC for finding a common fixed point of two nonexpansive mappings and compare them through a numerical example.

We take $T_{1}: R^{2} \rightarrow R^{2}$ by $T_{1}: v=\left(v_{1}, v_{2}\right)^{\top} \mapsto\left(\sin \frac{v_{1}+v_{2}}{\sqrt{2}}, \cos \frac{v_{1}+v_{2}}{\sqrt{2}}\right)^{\top}$ (see [9]) and $T_{2}: R^{2} \rightarrow R^{2}$ as $T_{2}:=P_{C}$ with $C=\left\{x \in R^{2}\|x-c\| \leq r\right\}$ where $c \in[-1,1]^{2}$ generated randomly, and $r=3$. The terminal condition is $\frac{\|x-T(x)\|+\|x-S(x)\|}{\|x\|} \leq \epsilon$. In the numerical results listed in the following table, 'Iter.' and 'Sec.' denote the number of iterations and the cpu time in seconds, respectively.

For randomly chosen initial values, we compare Algorithm 3.1 and Algorithm AC with different terminal condition many times, and results in the Table 1 were the average values. From Table 1 we observe that Algorithm 3.1 is better than Algorithm AC in the sense of the average.

Table 1: Numerical results for a given tolerance

\begin{tabular}{ccccc}
\hline \multirow{2}{*}{$x_{0}$} & \multicolumn{2}{c}{ Algorithm 3.1 } & \multicolumn{2}{c}{ Algorithm AC } \\
\cline { 2 - 5 } & Iter. & Sec. & Iter. & Sec. \\
\hline$\epsilon=0.01$ & 86 & 0.0234 & 114 & 0.0468 \\
\hline$\epsilon=0.001$ & 265 & 0.05772 & 322 & 0.07176 \\
\hline$\epsilon=0.0001$ & 559 & 0.11388 & 663 & 0.12168 \\
\hline
\end{tabular}

\section{Acknowledgements}

This work is supported by the National Natural Science Foundation of China (No. 61379102) and Fundamental Research Funds for the Central Universities which was approved in 2016, in part by the Foundation of Tianjin Key Lab for Advanced Signal Processing.

\section{References}

[1] P. K. Anh, C. V. Chung, Parallel hybrid methods for a finite family of relatively nonexpansive mappings, Numer. Func. Anal. Optim., 35 (2014), 649-664.1

[2] H. H. Bauschke, P. L. Combettes, Convex Analysis and Monotone Operator Theory in Hilbert Spaces, Springer, Berlin, (2011). 2.1, 2.3

[3] L. C. Ceng, N. C. Wong, J. C. Yao, Strong and weak convergence theorems for an infinite family of nonexpansive mappings and applications, Fixed Point Theory Appl., 2012 (2012), 21 pages.11

[4] Y. Censor, T. Elfving, A multiprojection algorithm using Bregman projections in a product space, Numer. Algorithms, 8 (1994), 221-239. 1

[5] Y. Censor, T. Elfving, N. Kopf, T. Bortfeld, The multiple-sets split feasibility problem and its applications for inverse problems, Inverse Problems, 21 (2005), 2071-2084.1

[6] J. P. Chancelier, Iterative schemes for computing fixed points of nonexpansive mappings in Banach spaces, J. Math. Anal. Appl., 353 (2009), 141-153.1

[7] Q. L. Dong, Y. Y. Lu, A new hybrid algorithm for a nonexpansive mapping, Fixed Point Theory Appl., 2015 (2015), 7 pages. 1

[8] Q. L. Dong, H. B. Yuan, Accelerated Mann and CQ algorithms for finding a fixed point of a nonexpansive mapping, Fixed Point Theory Appl., 2015 (2015), 12 pages.1]

[9] S. He, C. Yang, P. Duan, Realization of the hybrid method for Mann iterations, Appl. Math. Comput., 217 (2010), 4239-4247.4.

[10] K. Goebel, W. A. Kirk, Topics in Metric Fixed Point Theory, Cambridge Studies in Advanced Mathematics, Cambridge University Press, Cambridge, (1990).2.2

[11] C. Martinez-Yanes, H. K. Xu, Strong convergence of the CQ method for fixed point iteration processes, Nonlinear Anal., 64 (2006), 2400-2411.2.4

[12] K. Nakajo, W. Takahashi, Strong convergence theorems for nonexpansive mappings and nonexpansive semigroups, J. Math. Anal. Appl., 279 (2003), 372-379.1

[13] K. Nammanee, R. Wangkeeree, New iterative approximation methods for a countable family of nonexpansive mappings in Banach spaces, Fixed Point Theory Appl., 2011 (2011), 24 pages.1. 
[14] W. Nilsrakoo, S. Saejung, Weak and strong convergence theorems for countable Lipschitzian mappings and its applications, Nonlinear Anal., 69 (2008), 2695-2708.1

[15] M. V. Solodov, B. F. Svaiter, Forcing strong convergence of proximal point iterations in Hilbert space, Math. Program., 87 (2000), 189-202.3

[16] W. Takahashi, Viscosity approximation methods for countable families of nonexpansive mappings in Banach spaces, Nonlinear Anal., 70 (2009), 719-734.1.

[17] W. Takahashi, Y. Takeuchi, R. Kubota, Strong convergence theorems by hybrid methods for families of nonexpansive mappings in Hilbert spaces, J. Math. Anal. Appl., 341 (2008), 276-286.1.

[18] L. Wei, Y. J. Cho, H. Y. Zhou, A strong convergence theorem for common fixed points of two relatively nonexpansive mappings and its applications, J. Appl. Math. Comput., 29 (2009), 95-103.1

[19] Y. Yao, Y. C. Liou, N. C. Wong, Iterative algorithms based on the implicit midpoint rule for nonexpansive mappings, J. Nonlinear Convex A., (preprint).

[20] Y. Yao, M. Postolache, Y. C. Liou, Z. Yao, Construction algorithms for a class of monotone variational inequalities, Optim. Lett., (in press).

[21] H. Zhou, Y. Su, Strong convergence theorems for a family of quasi-asymptotic pseudo-contractions in Hilbert spaces, Nonlinear Anal., 70 (2009), 4047-4052.1. 